\title{
Isospin tracing: A probe of mixing and equilibration in heavy-ion
} collisions*

\author{
F. Rami ${ }^{\mathrm{a}}$ for the FOPI Collaboration \\ anstitut de Recherches Subatomiques, IN2P3-CNRS/ULP \\ 23, rue du Loess, F-67037 Strasbourg Cedex 2, France
}

The degree of equilibration attained in heavy-ion collisions is investigated using a new experimental approach which makes use of the isospin $(\mathrm{N} / \mathrm{Z})$ degree of freedom. The $\mathrm{N} /$ Z-ratio is used as a tracer to attribute the measured nucleons either to the target or to the projectile nucleons. Results for $\mathrm{Ru}(\mathrm{Zr})+\mathrm{Zr}(\mathrm{Ru})$ reactions at an incident energy of $400 \mathrm{~A} \mathrm{MeV}$ are reported. They show that the global equilibrium is not reached even in the most central collisions. Quantitative measures of stopping and mixing are obtained from the data. Comparisons are made with microscopic transport model calculations and information on the in-medium nucleon-nucleon elastic cross section is extracted

\section{INTRODUCTION}

The degree of projectile-target equilibration is one of the key issues in understanding the complex reaction mechanisms governing central heavy-ion collisions. This has profound implications on the theoretical concepts used to describe the collision process. It is still an open question whether the widely applied, at least local if not global, equilibrium assumption is valid [1,2], or whether significant non-equilibrium effects rather require the application of more elaborated non-equilibrium dynamical models [2-4]. This issue of equilibration is expected to be influenced by in-medium effects (such as Pauli blocking, Fermi motion) on the 'hard' scattering processes, by early 'soft' deflections in the momentum-dependent mean fields, and by finite-size (corona) effects. An understanding of all these effects is a prerequisite for a quantitative extraction of the equation-of-state (EOS) from nucleus-nucleus collisions.

At relativistic energies $(0.1$ to $2.0 \mathrm{~A} \mathrm{GeV})$, experimental investigations of the degree of equilibration were concentrated, up to now, on the measurement of the phase space distributions of the reaction products. Observables of interest were the width of rapidity distributions $[3,5]$ or the overall shape of the source [6,7]. The sensitivity of such observables is however reduced by effects like rescattering during the late phase of expansion.

In this contribution, we present a new experimental method allowing to explore directly, in a model independent approach, the degree of projectile-target equilibration achieved during the collision. The method relies on a new type of high precision measurement which makes use of the isospin $(N / Z)$ degree of freedom. The $N / Z$ is used as a tracer

\footnotetext{
*Invited talk given at NN2000 "Seventh International Conference on Nucleus- Nucleus Collisions", July 3-7, 2000, Strasbourg, France.
} 


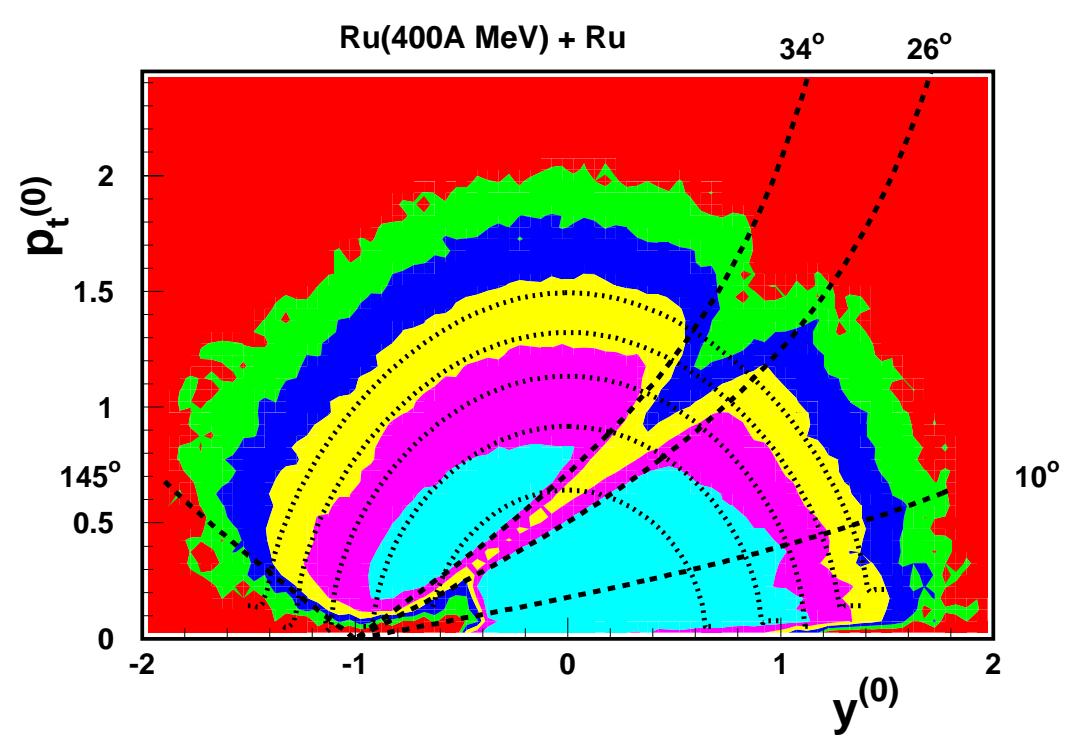

Figure 1. Invariant cross section in the $\left(y^{(0)}, p_{t}^{(0)}\right)$ plane of $Z=1$ particles measured in $\mathrm{Au}(400 \mathrm{~A} \mathrm{MeV})+\mathrm{Au}$ collisions under the $E_{\mathrm{RAT}}>1.4$ centrality cut. $y^{(0)}$ is the normalized rapidity, i.e. the particle c.m. rapidity divided by the projectile rapidity in the c.m. system. $p_{t}^{(0)}$ denotes the normalized transverse momentum, i.e. the particle transverse momentum per nucleon divided by the c.m. projectile momentum per nucleon. The grey levels correspond to different logarithmic cuts in the invariant cross section. See ref. [8] for more details.

to attribute the measured nucleons (on average) either to the target or to the projectile nucleons. The idea of using the $N / Z$ ratio to study the degree of mixing between projectile and target nucleons was investigated recently in microscopic transport calculations $[9,10]$. It was shown, in particular, that the degree of mixing is strongly sensitive to the value of the in-medium nucleon-nucleon $(\mathrm{n}, \mathrm{n})$ cross section used in the calculations. The new $N / Z$ tracer method has been applied in an experiment [8] performed recently with the FOPI apparatus, where all the four combinations of ${ }_{44}^{96} \mathrm{Ru}$ and ${ }_{40}^{96} \mathrm{Zr}$ nuclei, both as projectile and target, were measured at the same incident energy of $400 \mathrm{~A} \mathrm{MeV}$.

In Section 2, we will introduce the new method and the relevant experimental observables. Then, we will report (Section 3) on the observation of non-equilibrium effects in $\mathrm{Ru}+\mathrm{Zr}$ reactions and we will discuss (Section 4) the experimental results in the framework of the Isospin Quantum Molecular Dynamics (IQMD) model [11]. The main conclusions will be given in Section 5.

\section{THE $(N / Z)$-TRACER METHOD}

The method relies on the investigation of reactions between colliding nuclei with the same mass but different isospin content. We have measured all the four combinations of 
${ }_{44}^{96} \mathrm{Ru}$ and ${ }_{40}^{96} \mathrm{Zr}$ nuclei: ${ }_{44}^{96} \mathrm{Ru}$ on ${ }_{40}^{96} \mathrm{Zr},{ }_{40}^{96} \mathrm{Zr}$ on ${ }_{44}^{96} \mathrm{Ru},{ }_{44}^{96} \mathrm{Ru}$ on ${ }_{44}^{96} \mathrm{Ru}$ and ${ }_{40}^{96} \mathrm{Zr}$ on ${ }_{40}^{96} \mathrm{Zr}$. The choice of $\mathrm{Ru}$ and $\mathrm{Zr}$ isotopes takes advantage of an almost unique possibility offered by the periodic table of stable isotopes, while searching for two isobars of the largest possible $N / Z$ difference $^{2}$ which can be used both as projectile and target. The experiment $[8,12]$ was performed recently at the heavy-ion synchrotron SIS at GSI-Darmstadt using the FOPI apparatus. Details about the experimental conditions can be found in ref. $[13,14]$. The bombarding energy $(400 \mathrm{~A} \mathrm{MeV})$ was chosen at the minimum of the (n,n) cross section where the relative motion is significantly larger than the Fermi motion, but sufficiently low to avoid inelastic $(n, n)$ channels, while the $(n, n)$ angular distribution is almost isotropic.

Central collisions were selected in the experiment using the $E_{R A T}$ criterion $[6,7,12]$, i.e. the ratio of the sum of the transverse kinetic energies to the sum of the longitudinal kinetic energies. $E_{\mathrm{RAT}}$ is defined event wise as $E_{\mathrm{RAT}}=\sum_{\mathrm{i}=1}^{\mathrm{M}} E_{\mathrm{t}}^{\mathrm{i}} / \sum_{\mathrm{i}=1}^{\mathrm{M}} E_{1}^{\mathrm{i}}$, where $E_{\mathrm{t}}^{\mathrm{i}}$ and $E_{1}^{\mathrm{i}}$ are the transverse and longitudinal kinetic energies of the particle $\mathrm{i}$ and $\mathrm{M}$ is the number of detected particles. To isolate samples of highly central collisions, a condition requiring large $E_{R A T}$ values $\left(E_{\mathrm{RAT}}>1.4\right)$ was imposed to the measured events. This $E_{R A T}$ condition corresponds to about $1.5 \%$ of the total reaction cross section and to a geometrical impact parameter $b_{\text {geom }} \leq 1.3 \mathrm{fm}$ in a sharp-cut-off approximation. Fig.1 shows that the events selected under this $E_{\mathrm{RAT}}$ cut exhibit, in a representation of center-of-mass (c.m.) rapidity versus transverse momentum, a nearly isotropic source for the observed hydrogen $(Z=1)$ products. This is in agreement with previous observations $[5-7,15]$.

Let us now introduce the main idea of the $(N / Z)$-tracer method. Assume that we are observing the final number of protons, $Z$ in a given cell of the momentum space. The expected yield $Z^{\mathrm{Ru}}$ measured for the $\mathrm{Ru}+\mathrm{Ru}$ reaction is higher than $Z^{\mathrm{Zr}}$ of the $\mathrm{Zr}+\mathrm{Zr}$ reaction since $\mathrm{Ru}$ has 44 protons as opposed to 40 for Zr. Such measurements using identical projectile and target deliver calibration values $Z^{\mathrm{Ru}}$ and $Z^{\mathrm{Zr}}$ for each observed cell. In the case of a mixed reaction, $\mathrm{Ru}+\mathrm{Zr}$ or $\mathrm{Zr}+\mathrm{Ru}$, the measured proton yield $Z$ takes values intermediate between the calibration values $\left(Z^{\mathrm{Ru}}, Z^{\mathrm{Zr}}\right)$. If e.g. $Z$ is close to $Z^{\mathrm{Ru}}$ in a $\mathrm{Ru}+\mathrm{Zr}$ reaction, means that the cell is populated predominantly from nucleons of the Ru-projectile while if it is close to $Z^{\mathrm{Zr}}$ it is mostly populated from nucleons of the Zr-target. In this way it is possible to trace back the relative abundance of target to projectile nucleons contributing to a given cell.

The following definition for the relative abundance of the projectile-target nucleons has been adopted:

$R_{\mathrm{Z}}=\frac{2 \times Z-Z^{\mathrm{Zr}}-Z^{\mathrm{Ru}}}{Z^{\mathrm{Zr}}-Z^{\mathrm{Ru}}}$

By definition the ratio $R_{\mathrm{Z}}$ takes +1 for $\mathrm{Zr}+\mathrm{Zr}$ and -1 for $\mathrm{Ru}+\mathrm{Ru}$. Fig.2 illustrates the expected dependences of $R_{Z}$ as a function of the c.m. rapidity. In the case of full equilibrium in a mixed reaction, $R_{\mathrm{Z}}$ would be equal to 0 (equal number of projectile and target nucleons) everywhere independently of the location of the cell in the phase space. Note that 'equilibrium' means here the complete mixing of projectile and target nucleons. In a transparency scenario the value of $R_{\mathrm{Z}}$ for $\mathrm{Zr}$ (beam) on $\mathrm{Ru}$ (target) is expected to be positive in the forward $\left(y^{(0)}>0\right)$ c.m. hemisphere and negative in the backward $\left(y^{(0)}<0\right)$ c.m. hemisphere; while for the other mixed reaction $\mathrm{Ru}$ (beam) on $\mathrm{Zr}$ (target)

${ }^{2}$ The $N / Z$ ratio is equal to 1.18 and 1.40 for ${ }_{44}^{96} \mathrm{Ru}$ and ${ }_{40}^{96} \mathrm{Zr}$, respectively. 


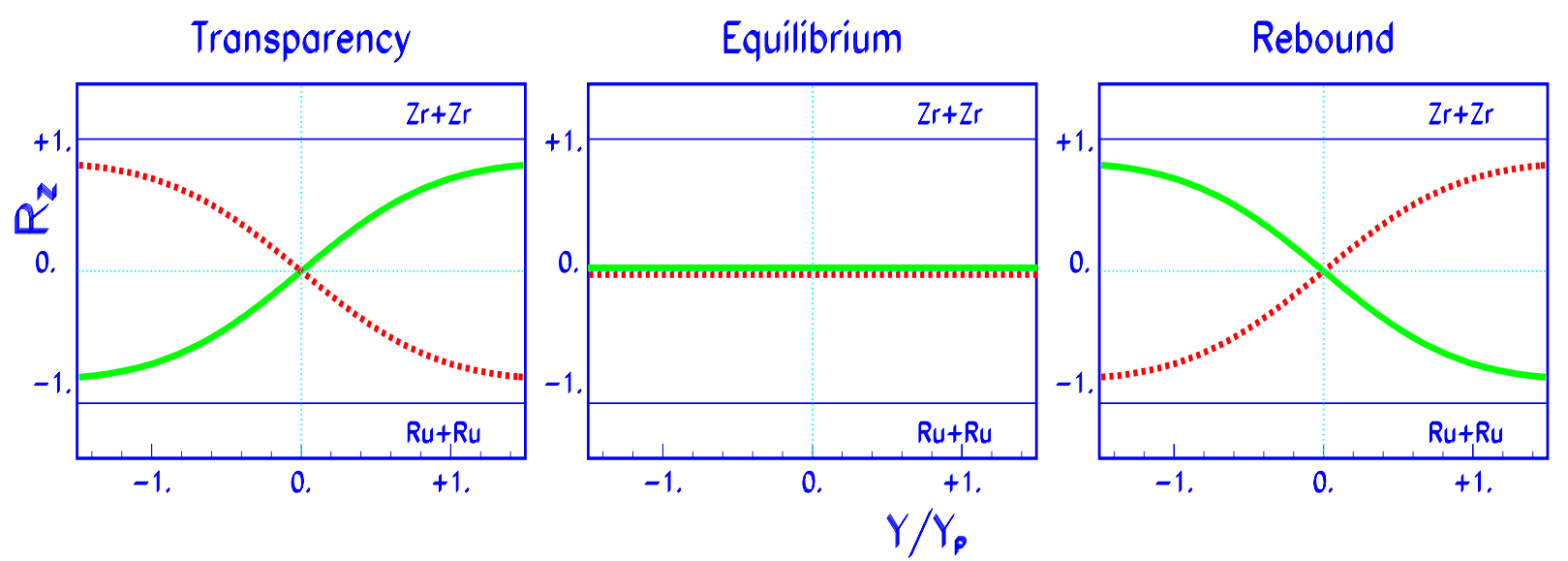

Figure 2. Relative abundance $R_{\mathrm{Z}}$ as a function of the normalized c.m. rapidity. The curves illustrate the trends expected for the mixed reactions: $\mathrm{Ru}+\mathrm{Zr}$ (dashed) and $\mathrm{Zr}+\mathrm{Ru}$ (full). Three different scenarios are shown: transparency, equilibrium and rebound.

one expects negative values in the forward region and positive values in the backward region. A rebound scenario would correspond to an inverted situation as compared to the one described above for transparency.

There are several advantages in using the $(N / Z)$-tracer method:

- The four reaction combinations are investigated, under identical experimental conditions so that the ratios are insensitive to systematic uncertainties due to the apparatus. The errors are essentially of statistical nature and profit from the high yield in the considered cell.

- The mixed reaction $\mathrm{Zr}+\mathrm{Ru}$ is the same as $\mathrm{Ru}+\mathrm{Zr}$ except that target and projectile are inverted: this allows forward-backward cross-checks of the apparatus which in addition can also be obtained from the symmetric $\mathrm{Ru}+\mathrm{Ru}$ and $\mathrm{Zr}+\mathrm{Zr}$ reactions.

- Using the four reactions the full information needed can be obtained by measuring only within the c.m. backward or only the c.m. forward hemisphere.

\section{NON-EQUILIBRIUM EFFECTS: WHAT CAN WE LEARN FROM THE EXPERIMENT ?}

Fig. 3 shows the relative abundance $R_{\mathrm{Z}}$ extracted from the data in the case of highly central collisions $\left(E_{\mathrm{RAT}}>1.4\right.$ ). The $R_{\mathrm{Z}}$-ratio is obtained according to equation (1) with $Z$ being the sum of the number of detected free protons plus the number of protons detected within the deuterons. The experimental results are presented in the backward c.m. hemisphere where the background contribution (due to the oxygen content of the target) is negligible [8]. As can be seen in Fig.3, the inversion of projectile and target nuclei changes, as expected, the sign of the $R_{\mathrm{Z}}$-ratio and the values obtained for both mixed 
Figure 3. Relative abundance $R_{Z}$ as a function of the normalized c.m. rapidity $y^{(0)}$. The experimental results are shown for both isospin asymmetric reactions $\mathrm{Ru}+\mathrm{Zr}$ (full circles) and $\mathrm{Zr}+\mathrm{Ru}$ (open circles) in the case of highly central collisions. The error bars correspond to statistical uncertainties. The horizontal dashed lines indicate the values of $R_{Z}=$ +1 and -1 corresponding to the isospin symmetric reactions $\mathrm{Zr}+\mathrm{Zr}$ and $\mathrm{Ru}+\mathrm{Ru}$, respectively.

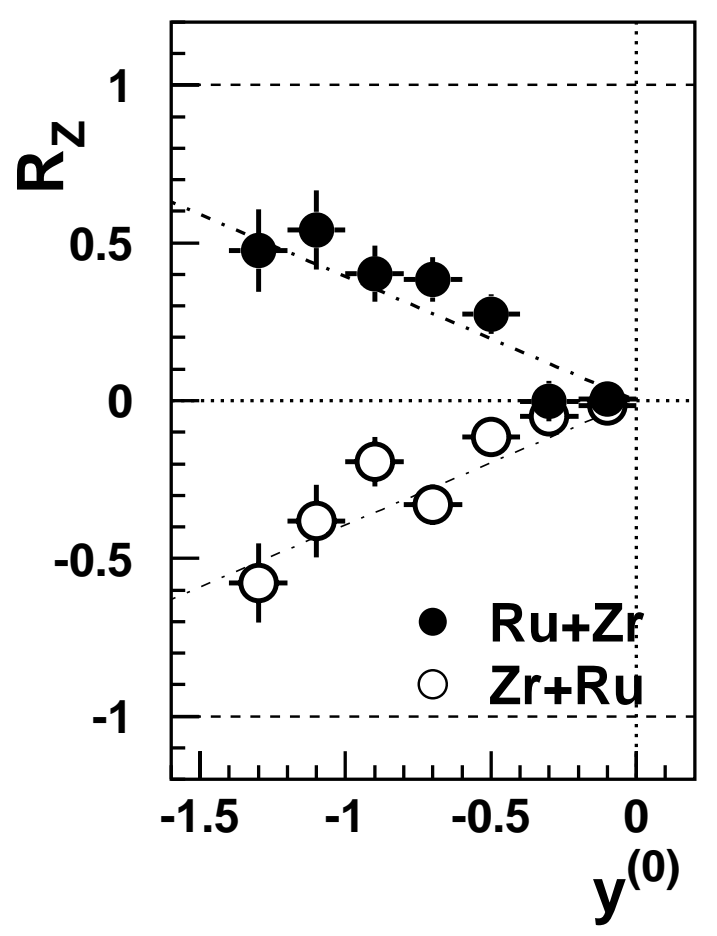

reactions are consistent within errors. It is interesting to note also that at $y^{(0)}=0$, one observes for both mixed reactions a value of $R_{Z}$ close to 0 as expected in this rapidity region where projectile and target nucleons must be completely mixed. All these observations accredit the reliability of the $(N / Z)$-tracer method and its sensitivity.

The results presented in Fig.3 allow us to map the degree of mixing between projectile and target nucleons across a large region of the phase space. They show clearly a substantial departure from the expected trend for a complete mixing scenario (indicated by the horizontal dotted line). The data are consistent with the presence of sizeable transparency effects.

This evidence for non-equilibrium effects is also observed in Fig.4a which shows the centrality dependence of the $R_{\mathrm{Z}}$-ratio. Here, data are presented within a wide c.m. rapidity range: $-0.75 \leq y^{(0)} \leq-0.25$. The impact parameter $b_{\text {geom }}$ is derived by integrating over the measured cross section as a function of $E_{\mathrm{RAT}}$ or of the charged particle multiplicity. The observed trend corroborates the picture of an incomplete mixing between projectile and target nucleons which persists up to the end of the collision, an observation that holds even for the most central collisions, for $b_{\text {geom }} \rightarrow 0$. Note on the other hand that, as expected, the magnitude of transparency effects increases with increasing impact parameters. 

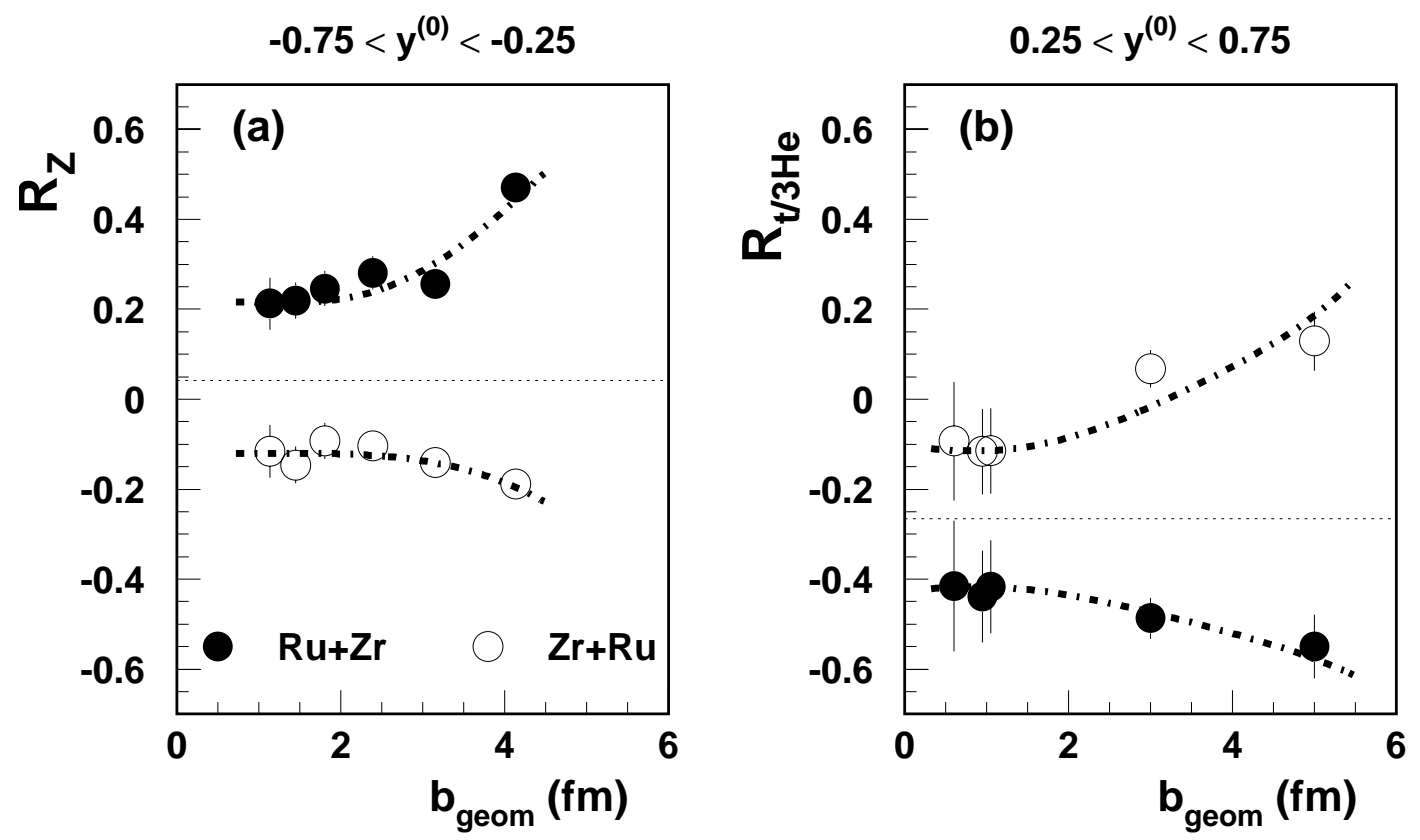

Figure 4. $R_{\mathrm{Z}}$ (left panel) and $R_{\mathrm{t} /{ }^{3} \mathrm{He}}$ (right panel) as a function of the geometrical impact parameter. The horizontal dotted lines correspond to the values $\left(R_{\mathrm{Z}}=0.04\right.$ and $R_{\mathrm{t} /{ }^{3} \mathrm{He}}=$ -0.27 ) at halfway between the $\mathrm{Ru}+\mathrm{Zr}$ and $\mathrm{Zr}+\mathrm{Ru}$ experimental points in the $b_{\text {geom }} \leq$ $1.5 \mathrm{fm}$ region. The dotted-dashed lines are just to guide the eye.

Similar conclusions can be also drawn from the inspection of Fig.4b. Here the relative abundance ratio is extracted from another observable, the ratio of tritium to ${ }^{3} \mathrm{He}\left(\mathrm{t} /{ }^{3} \mathrm{He}\right)$, which is also sensitive to the $N / Z$ ratio [16]. The t $/{ }^{3} \mathrm{He}$ ratio was measured in the forward c.m. hemisphere where a good time-of-flight particle identification is achieved in the FOPI experiment $[13,14]$. Here, the quantity $R_{\mathrm{t} /{ }^{3} \mathrm{He}}$ is defined in a similar way as for $R_{\mathrm{Z}}$, using equation (1) with $Z$ being replaced by the $\mathrm{t} /{ }^{3} \mathrm{He}$ ratio. Except for an off-set of $R_{\mathrm{t} /{ }^{3} \mathrm{He}}=-0.27 \pm 0.07$ for the $\mathrm{t} /{ }^{3} \mathrm{He}$-ratio, one observes qualitatively the same trend as for the $R_{\mathrm{Z}}$-ratio. Such an off-set can be understood from a non-linear dependence of the $\mathrm{t} /{ }^{3} \mathrm{He}$ ratio as a function of isospin. We find that, for $N>Z$, an empirical dependence of the $\mathrm{t} /{ }^{3} \mathrm{He}$ ratio of $\left[1+100 \times\left(\frac{N-Z}{N+Z}\right)^{2.5}\right]$ describes the known systematics [17] and satisfies the measured off-set of -0.27 .

Fig. 5 displays the overall rapidity-density distribution $\mathrm{d} N / \mathrm{d} y^{(0)}$ (circles) measured in central $\mathrm{Ru}+\mathrm{Ru}$ collisions. This distribution was obtained [16] by extrapolating the measured transverse momentum spectra, within the backward detector acceptance, using the thermal blast model proposed by Siemens and Rasmussen [18]. The forward part of the distribution (open circles) was deduced by assuming a backward/forward symmetry.

As stated before, the $R_{\mathrm{Z}}$-ratio gives a measure of the relative abundance of projectile and target nucleons. This can be used to deconvolute the overall rapidity distribution into separated distributions for the projectile and for the target nucleons. For this purpose 


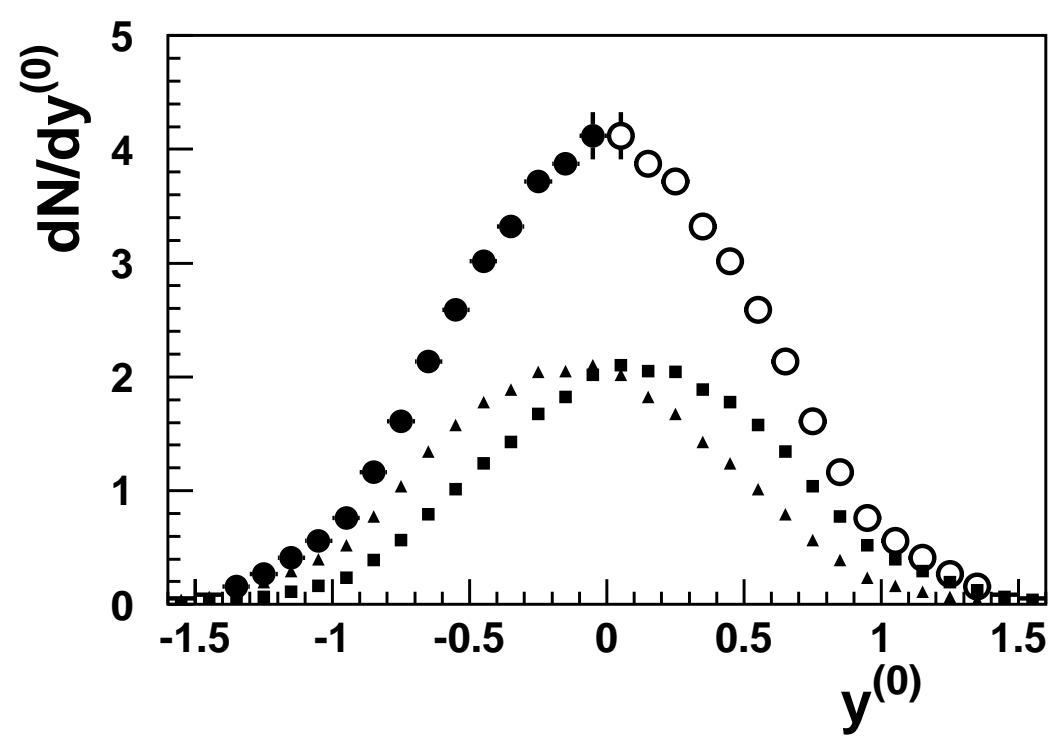

Figure 5. Experimental c.m. rapidity distribution (circles) of protons, free plus those detected within the deuterons, in central $\mathrm{Ru}+\mathrm{Ru}$ collisions. Data points in the forward region (open circles) were obtained by assuming a backward/forward symmetry. The two other distributions have been obtained by unfolding the overall distribution into 'projectile' (squares) and 'target' (triangles) components.

we use the dashed-dotted line $\left( \pm 0.393 y^{(0)}\right)$ in Fig.3 which describes an average of both measurements for $\mathrm{Ru}+\mathrm{Zr}$ and $\mathrm{Zr}+\mathrm{Ru}$ reactions. For each rapidity bin, the number of projectile (target) nucleons is obtained as $N_{\mathrm{pr}}=0.5\left(1+0.393 y^{(0)}\right) N\left(N_{\mathrm{tr}}=0.5(1-\right.$ $\left.0.393 y^{(0)}\right) N$ ).

After deconvolution of the overall $\mathrm{d} N / \mathrm{d} y^{(0)}$ distribution, a shift between the two deduced rapidity distributions (see Fig.5) emerges, demonstrating that a memory of the initial target-projectile translatory motion survives throughout a central collision. Transparency effects are clearly visible here with more projectile nucleons in the forward c.m. hemisphere and more target nucleons in the backward c.m. hemisphere.

In order to characterize with few numbers the information contained in the separated projectile (or target) distribution, we define the following quantities:

- A shift $\left\langle y_{\mathrm{pr}}^{(0)}\right\rangle$ of the mean value with respect to mid-rapidity; $\left\langle y_{\mathrm{pr}}^{(0)}\right\rangle=0$ corresponds to full stopping and full thermo/chemical equilibrium, while $\left\langle y_{\mathrm{pr}}^{(0)}\right\rangle=1$ corresponds to the initial projectile rapidity without any stopping; positive values of $\left\langle y_{\mathrm{pr}}^{(0)}\right\rangle$ are expected for transparency, negative values for a backward rebound of the projectile nucleons from the target.

- A mixing value $M_{\mathrm{pr}}=\left(N_{f}-N_{b}\right) /\left(N_{f}+N_{b}\right)$, where $N_{f}$ is the number of projectile nucleons emitted forward and $N_{b}$ backwards; $M_{\mathrm{pr}}$ is a measure of non-equilibrium effects.

- A width of the unfolded distribution, $\sigma_{\mathrm{pr}}$. 


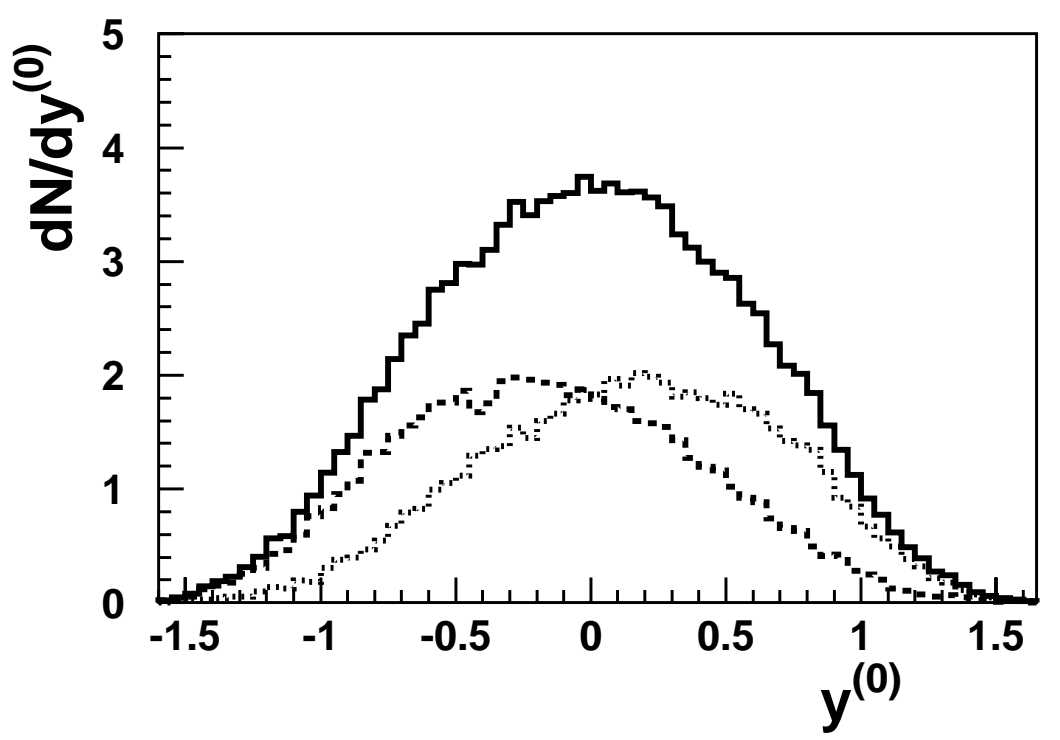

Figure 6. Calculated c.m. rapidity distribution (solid histogram) of nucleons emitted in central $(b \leq 1 \mathrm{fm}) \mathrm{Ru}(400 \mathrm{~A} \mathrm{MeV})+\mathrm{Ru}$ collisions. The two other distributions have been obtained by tagging projectile (dotted) and target (dashed) nucleons in the model.

These quantities provide a quantitative information of the strength of the non-equilibrium effects. This is, as we will see in the next section, very convenient in order to discuss the experimental results within the framework of theoretical models.

\section{DYNAMICAL MODEL CALCULATIONS: CONSTRAINTS ON THE IN- MEDIUM NUCLEON-NUCLEON CROSS SECTION}

We compare here the data presented in the previous section to the predictions of the IQMD-model [11]. The latter is a version of the Quantum Molecular Dynamics (QMD) model $[1,3]$ which takes into account isospin degrees of freedom into the cross sections and the Coulomb interaction. Calculations were made using a stiff EOS, with a compressibility coefficient $K=380 \mathrm{MeV}$, and momentum dependent interactions (MDI). The impact parameter was limited to $b \leq 1 \mathrm{fm}$, a condition similar to the centrality selection applied to the data.

In Fig. 6 we show the rapidity-density distributions predicted for the $\mathrm{Ru}(400 A \mathrm{MeV})+\mathrm{Ru}$ reaction. The two separated distributions (indicated by the dotted and dashed curves) were obtained here by tagging the projectile and target nucleons in the calculations. As can be seen in the figure, the model also predicts a sizeable transparency: there is no complete overlap of the projectile and rapidity distributions. The predictions are in qualitative agreement with the data (Fig.5). 


\begin{tabular}{cccc}
\hline \hline & tagged & $N / Z$-tracer & $N / Z$-tracer + filter \\
\hline$\left\langle y_{\mathrm{pr}}^{(0)}\right\rangle$ & 0.16 & 0.15 & 0.15 \\
$\sigma_{\mathrm{pr}}$ & 0.55 & 0.55 & 0.56 \\
$M_{\mathrm{pr}}$ & 0.23 & 0.22 & 0.21 \\
\hline \hline
\end{tabular}

Table 1

Mean value $\left\langle y_{\mathrm{pr}}^{(0)}\right\rangle$, width $\sigma_{\mathrm{pr}}$ of the projectile rapidity distribution (in $y^{(0)}$ units) and mixing observable $M_{\mathrm{pr}}$ calculated for the $R u+R u$ reaction using the IQMD model (see text for more details).

Before going to more quantitative comparisons with the data, it is interesting to examine, within the model, the reliability of the $(N / Z)$-tracer method. To this end, we have compared the values obtained by applying the $(N / Z)$-tracer method to those extracted directly from the projectile rapidity distribution obtained by tagging the nucleons in the model. The results are given in Table 1 for the three observables, $\left\langle y_{\mathrm{pr}}^{(0)}\right\rangle, \sigma_{\mathrm{pr}}$ and $M_{\mathrm{pr}}$, defined in Section 3. As can be seen, both calculations lead to very similar results. This confirms clearly the validity of the $(N / Z)$-tracer method. Moreover, the effect of the detector filter (last column in Table 1) is found to be negligible. This results from the fact that the method relies on relative quantities.

\begin{tabular}{ccccccc}
\hline \hline & & \multicolumn{4}{c}{ IQMD } \\
\cline { 3 - 7 } & data & HM & HM & HM & H & SM \\
& & $\sigma_{\mathrm{nn}}^{\text {free }}$ & $0.5 \times \sigma_{\mathrm{nn}}^{\text {free }}$ & $1.2 \times \sigma_{\mathrm{nn}}^{\text {free }}$ & $\sigma_{\mathrm{nn}}^{\text {free }}$ & $\sigma_{\mathrm{nn}}^{\text {free }}$ \\
\hline$\left\langle y_{\mathrm{pr}}^{(0)}\right\rangle$ & 0.11 & 0.16 & 0.33 & 0.11 & 0.10 & 0.16 \\
$\sigma_{\mathrm{pr}}$ & 0.52 & 0.55 & 0.59 & 0.54 & 0.52 & 0.56 \\
$M_{\mathrm{pr}}$ & 0.17 & 0.23 & 0.43 & 0.16 & 0.15 & 0.22 \\
\hline \hline
\end{tabular}

Table 2

Comparison between data and different IQMD model predictions. Systematic errors on the values of $\left\langle y_{\mathrm{pr}}^{(0)}\right\rangle$ and $M_{\mathrm{pr}}$ extracted from the data are about 10\%. IQMD calculations are obtained by tagging projectile and target nucleons in the model. HM stands for a hard equation-of-state and momentum dependent interaction; $H$ stands for a hard equation-of-state without momentum dependent interaction and SM stands for a soft equation-of-state $(K=200 \mathrm{MeV})$ and momentum dependent interaction. $\sigma_{\mathrm{nn}}^{\text {free }}$ is the free $(n, n)$ cross section (default value in the model).

Calculations using different model parameters are presented in Table 2 together with the experimental results. As can be seen, both observables $\left\langle y_{\mathrm{pr}}^{(0)}\right\rangle$ and $M_{\mathrm{pr}}$ exhibit a quite strong sensitivity to the in-medium $(n, n)$-cross section $\left(\sigma_{n n}^{\text {med }}\right)$ used in the calculations. A reduction of $50 \%$ of the $(n, n)$-cross section $\left(\sigma_{\mathrm{nn}}^{\text {med }}=0.5 \times \sigma_{\mathrm{nn}}^{\text {free }}\right)$ leads to an increase of both $\left\langle y_{\mathrm{pr}}^{(0)}\right\rangle$ and $M_{\mathrm{pr}}$ by about a factor of 2 . This strong sensitivity to $\sigma_{\mathrm{nn}}^{\text {med }}$ was also 


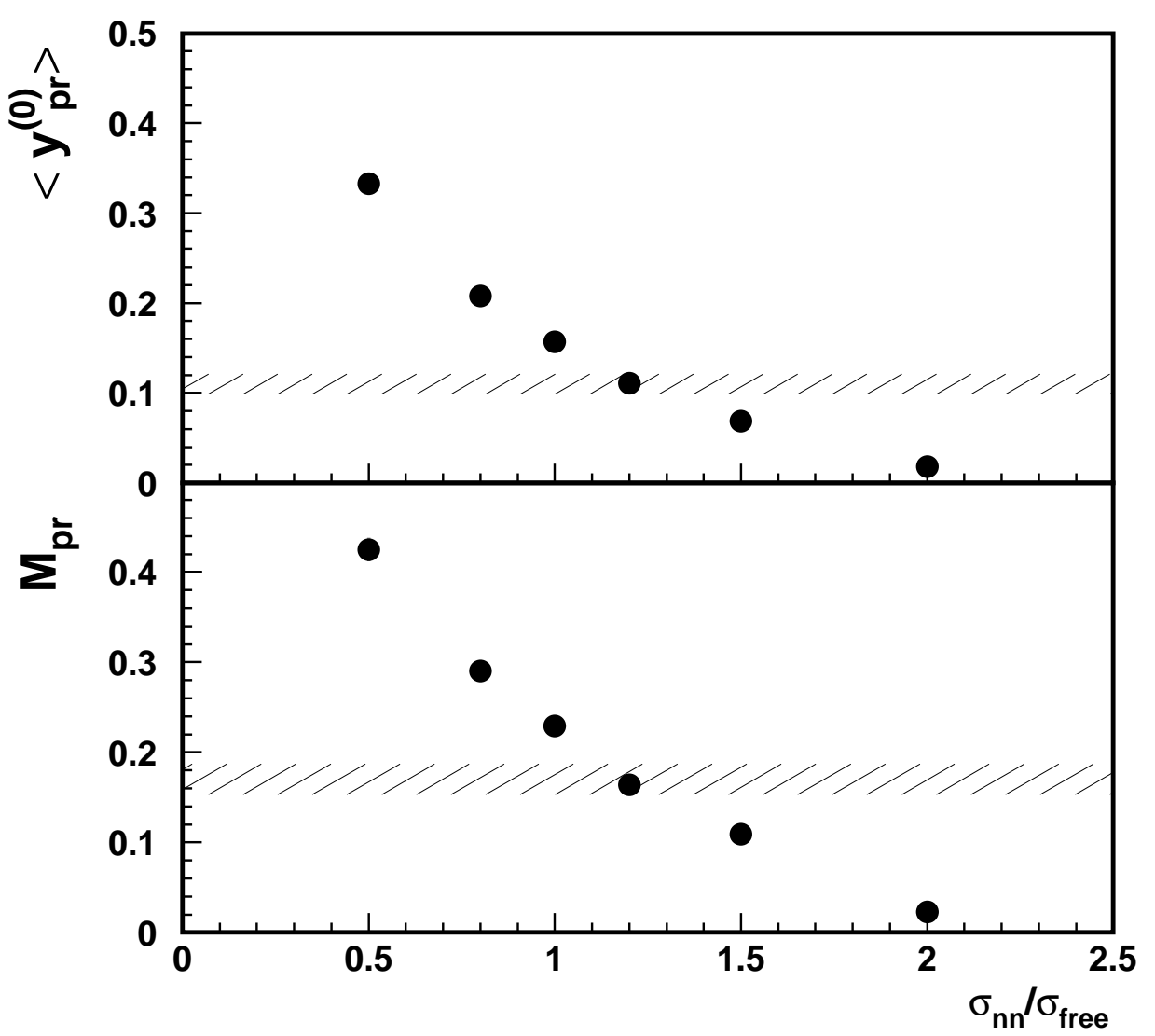

Figure 7. Rapidity shift $\left\langle y_{\mathrm{pr}}^{(0)}\right\rangle$ and mixing observable $M_{\mathrm{pr}}$ as a function of the value of the in-medium n-n cross section used in the IQMD model. The in-medium n-n cross section $\sigma_{\mathrm{nn}}$ is normalized to the free n-n cross section $\sigma_{\mathrm{nn}}^{\text {free }}$ ). The calculations (full circles) are compared to the experimental results (indicated by the horizontal hatched areas).

reported recently in the framework of different microscopic transport approaches $[9,10]$. It is also interesting to notice in Table 2 that MDI effects act against equilibration: HM parameterization gives larger values of $\left\langle y_{\mathrm{pr}}^{(0)}\right\rangle$ and $M_{\mathrm{pr}}$ as compared to those obtained with the $\mathrm{H}$ parametrization. On the other hand, the influence of the stiffness of the EOS is found to be very weak: HM and SM calculations lead, within statistical uncertainties, to the same results.

The best agreement with the data is obtained for a value of $\sigma_{\mathrm{nn}}^{\text {med }}$ slightly higher, by about $20 \%$, than the free $(\mathrm{n}, \mathrm{n})$-cross section $\left(\sigma_{\mathrm{nn}}^{\text {free }}\right)$. This is clearly visible in Fig. 7 which shows the dependence of both observables $\left\langle y_{\mathrm{pr}}^{(0)}\right\rangle$ and $M_{\mathrm{pr}}$ on the in-medium n-n cross section. This result indicates that, within this model, a significant modification of the $(n, n)$-cross section in the nuclear medium is to be excluded. 


\section{SUMMARY AND OUTLOOK}

In this contribution, we have reported on a new experimental method to investigate the degree of equilibration reached in central heavy-ion collisions. The proposed method relies on a new type of high precision measurement which makes use of the isospin degree of freedom. This idea has been applied in an experiment performed recently with the FOPI apparatus, where all the four combinations of ${ }_{44}^{96} \mathrm{Ru}$ and ${ }_{40}^{96} \mathrm{Zr}$ nuclei, both as projectile and target, were measured at the same incident energy of $400 \mathrm{~A} \mathrm{MeV}$.

We have shown that this method allows the relative abundance between projectile and target nucleons to be mapped across a large domain of the phase space. The results clearly indicate that the global equilibrium is not reached even in the most central collisions. They are consistent with the presence of sizeable transparency effects. This evidence for non-equilibrium effects has been observed from the analysis of two different experimental observables: the number of measured protons and the $\mathrm{t} /{ }^{3} \mathrm{He}$ ratio.

The $(N / Z)$-tracer method allowed us to deconvolute the experimental rapidity-density distribution $\mathrm{d} N / \mathrm{d} y^{(0)}$ into separated distributions for the projectile and for the target nucleons. Quantitative measures of stopping and mixing were then extracted from the separated rapidity distributions and the obtained values were compared to different predictions of the IQMD model. The best agreement between data and model calculations was found for a value of the (n,n)-cross section slightly higher, by about $20 \%$, than the free $(n, n)$-cross section. This indicates that, within this model, a significant modification of the $(n, n)$-cross section in the nuclear medium is to be excluded.

In conclusion, the proposed $N / Z$-tracer method exhibits a high sensitivity to the degree of mixing between projectile and target nucleons. This opens a new channel for the study of non-equilibrium features in heavy-ion collisions. It would be very interesting to see, for example, how non-equilibrium effects evolve with the incident energy [10,19]. Also, the use of radioactive beams could enhance significantly the sensitivity of the method and should be therefore considered in future experiments.

This work was supported in part by the French-German agreement between GSI and IN2P3/CEA (Project No.97-29).

\section{REFERENCES}

1. H. Stöcker and W. Greiner, Phys. Rep. 137 (1986) 277.

2. G. F. Bertsch, H. Kruse and S. Das Gupta, Phys. Rev. C 32 (1984) R673.

3. J. Aichelin, Phys. Rep. 202 (1991) 233.

4. H. Feldmeier, Nucl. Phys. A 515 (1990) 147.

5. B. Hong et al., FOPI Collaboration, Phys. Rev. C 57 (1998) 244.

6. S. C. Jeong et al., FOPI Collaboration, Phys. Rev. Lett. 72 (1994) 3468.

7. W. Reisdorf et al., FOPI Collaboration, Nucl. Phys. A 612 (1997) 493.

8. F. Rami, FOPI Collaboration, Phys. Rev. Lett. 84 (2000) 1120.

9. S. A. Bass et al., Prog. Part. Nucl. Phys. 41 (1998) 225.

10. A. Hombach, W. Cassing and U. Mosel, Eur. Phys. J. A 5, (1999) 77.

11. C. Hartnack et al., Eur. Phys. J. A 1, (1998) 151.

12. B. de Schauenburg, Ph-D thesis, Strasbourg (1999), IReS 99-06. 
13. A. Gobbi et al., FOPI Collaboration, Nucl. Inst. Meth. A 324 (1993) 156.

14. J. Ritman et al., FOPI Collaboration, Nucl. Phys. (Proc. Suppl.) B 44 (1995) 708.

15. M. A. Lisa, EOS Collaboration, Phys. Rev. Lett. 75 (1995) 2662.

16. Y. Leifels et al., FOPI Collaboration, GSI Scientific Report 1997, GSI 98-1 (1998) 57.

17. S. Nagamiya et al., Phys. Rev. C 24 (1981) 971.

18. P.J. Siemens and J.O. Rasmussen, Phys. Rev. Lett. 42 (1979) 880.

19. R. Nebauer and J. Aichelin, Proceedings of the " $3^{\text {rd }}$ Catania Relativistic Ion Studies", Acicastello, Italy, May22-26, 2000, to appear in Nucl.Phys. A. 